\title{
Nonnegative Trigonometric Polynomials and Certain Rational Characteristic Functions
}

\author{
Eugene Lukacs ${ }^{1}$ and Otto Szász ${ }^{2}$
}

\begin{abstract}
Let $0<b_{1}<b_{2}<\ldots<b_{n}$ be $n$ integers and $0<d_{1}<d_{2}<\ldots<d_{m}$ be $m(m \leqq n)$ real numbers (not necessarily integers). Denote by $g(\theta)$ the Vandermonde determinant formed from the $b_{1}^{2}, b_{2}^{2}, \ldots, b_{n}^{2}$ with the first row replaced by $1-\lambda_{j} \cos b_{j} \theta$, where $\lambda_{j}=\prod_{k=1}^{m}\left(1-b_{j}^{2} / d_{k}^{2}\right)$ for $j=1,2, \ldots, n$. The question whether $g(\theta)$ is nonnegative for all values of $\theta$ is closely connected with the problem whether certain rational functions are characteristic functions. Four configurations of the $b_{1}, \ldots, b_{n}$, and $d_{1}, \ldots, d_{m}$ are studied, which lead to nonnegative trigonometric polynomials.
\end{abstract}

\section{Introduction}

In this paper we study several nonnegative trigonometric polynomials that are connected with the question whether certain rational functions are Fourier transforms of distribution functions (characteristic functions).

We consider in the following a rational function $\phi(t)$ whose poles and zeros have the same imaginary part $a$ and assume further that all poles and all zeros have the order one. Let $-i a, i v_{j},-i \bar{v}_{j}(j=1,2, \ldots, n)$ be the poles and $-i \bar{w}_{k},-i w_{k}(k=1,2, \ldots, m), m \leq n$, be the zeros of $\phi(t)$, where $v_{j}=a+i b_{j}, w_{k}=a+i d_{k}$, and $a>0,0<b_{1}<b_{2}<\ldots<b_{n}, 0<d_{1}<d_{2}<\ldots<d_{m}$. Such a function $\phi(t)$ fulfills the necessary conditions that a rational function must satisfy in order to be the Fourier transform of a distribution function. These conditions were given in two earlier papers $\lfloor 1,2] .^{3}$

In the following we denote by

$$
V_{k}=\left|\begin{array}{llll}
b_{1}^{2 k}, & b_{2}^{2 k}, & \ldots & b_{n}^{2 k} \\
b_{1}^{2}, & b_{2}^{2}, & \ldots & b_{n}^{2} \\
\cdot & \cdot & \ldots & . \\
\cdot & \cdot & \cdots & \cdot \\
\cdot & \cdot & \cdots & \cdot \\
b_{1}^{2(n-1)}, & b_{2}^{2(n-1)}, & \ldots & b_{n}^{2(n-1)}
\end{array}\right|
$$

the generalized Vandermonde determinant formed from the numbers $b_{1}^{2}, \ldots, b_{n}^{2}$ with the exponents $k, 1,2, \ldots,(n-1)$. We write further $\Delta_{j}$ for the minor of the element in the first row and $j$ th column of this determinant, so that $V_{k}=\sum_{j=1}^{n}(-1)^{j-1} b_{j}^{2 k} \Delta_{j}$.

In order to decide whether the function $\phi(t)$ is a characteristic function, we decompose it into partial fractions and apply the Fourier inversion formula. We see then after some computation that $\phi(t)$ is the Fourier transform of a certain function $f(x)$, i. e., $\phi(t)=\int_{-\infty}^{\infty} e^{i t x} f(x) d x$, where $f(x)$ is given by

$$
f(x)= \begin{cases}0, & \text { if } x<0 \\ C e^{-a x}\left\{V_{0}-\sum_{j=1}^{n}(-1)^{j-1} \Delta_{j} \lambda_{j} \cos b_{j} x\right\}, & \text { if } x>0\end{cases}
$$

${ }^{1}$ National Bureau of Standards.

${ }^{2}$ University of Cincinnati. Deceased September 19, 1952.

${ }^{3}$ Figures in brackets indicate the literature references at the end of this paper. 
with

$$
\begin{gathered}
C=\frac{a \prod_{j=1}^{n}\left(a^{2}+b_{j}^{2}\right) \prod_{k=1}^{m} d_{k}^{2}}{V_{0} \prod_{k=1}^{m}\left(a^{2}+d_{k}^{2}\right) \prod_{j=1}^{n} b_{j}^{2}} \\
\lambda_{j}=\prod_{k=1}^{m}\left(1-\frac{b_{j}^{2}}{d_{k}^{2}}\right) \quad(j=1,2, \ldots n) .
\end{gathered}
$$

If we introduce the polynomial

$$
H(z)=c_{0}\left(z-d_{1}^{2}\right)\left(z-d_{2}^{2}\right) \ldots\left(z-d_{m}^{2}\right)=\sum_{k=0}^{m} c_{k} z^{m-k},
$$

where

$$
c_{0}=(-1)^{m}\left\{\prod_{k=1}^{m} d_{k}^{2}\right\}^{-1} \text { and } c_{m}=1,
$$

we can write

$$
\lambda_{j}=H\left(b_{j}^{2}\right), \quad j=1,2, \ldots, \mathrm{n} .
$$

The function $f(x)$ as determined by (2) is real, after some computation it is seen that $\int_{0}^{\infty} f(x) d x=1$. If $f(x)$ is nonnegative, it is the frequency function of an absolutely continuous probability distribution. We obtain therefore the following condition:

Condition I. The rational function $\phi(t)$ is a characteristic function if, and only if,

for all $\theta$.

$$
g(\theta)=\left|\begin{array}{cccc}
\left(1-\lambda_{1} \cos b_{1} \theta\right), & \left(1-\lambda_{2} \cos b_{2} \theta\right), & \ldots & \left(1-\lambda_{n} \cos b_{n} \theta\right) \\
b_{1}^{2}, & b_{2}^{2}, & \ldots & b_{n}^{2} \\
\cdot & \cdot & \cdots, & \cdot \\
\cdot & \cdot & \cdots & \cdot \\
\cdot & \cdot & \cdots & \cdot \\
b_{1}^{2(n-1)}, & b_{2}^{2(n-1)}, & \ldots, & b_{n}^{2(n-1)}
\end{array}\right| \geq 0
$$

If we consider a rational function without zeros, that is, if we assume that $\phi_{0}(t)$ is the reciprocal of a polynomial whose roots are $-i a,-i v_{j},-i \bar{v}_{j}(j=1,2, \ldots, n)$, then we derive by a similar reasoning the following statement:

Condition II. The function $\phi_{0}(t)$ is a characteristic function if, and only if,

for all $\theta$.

$$
g_{0}(\theta)=\left|\begin{array}{cccc}
1-\cos b_{1} \theta, & 1-\cos b_{2} \theta, & \ldots & 1-\cos b_{n} \theta \\
b_{1}^{2}, & b_{2}^{2}, & \ldots, & b_{n}^{2} \\
\cdot & \cdot & \ldots & . \\
\cdot & \cdot & \ldots & \cdot \\
\cdot & \cdot & \cdots, & \cdot \\
b_{1}^{2(n-1)}, & b_{2}^{2(n-1)}, & \ldots, & b_{n}^{2(n-1)}
\end{array}\right| \geq 0
$$

This condition is analogous to (5), in fact, (6) may be obtained from (5) by putting $\lambda_{j}=1$ for $j=1,2, \ldots, n$. We agree, therefore, to put $\lambda_{j}=1$ whenever $m=0$.

In this paper we consider the determinant (5) only for integer values of the $b_{j}$ and show that it represents for certain configurations of the $b_{j}$ and $d_{k}$ a nonnegative trigonometric polynomial. In this manner we can construct certain rational characteristic functions. 


\section{Some Lemmas}

For the discussion of the nonnegativity of $g(\theta)$ we need the following lemmas:

Lemma 1. If $b_{1}, b_{2}, \ldots, b_{n}$ are integers, the determinant (5) can be factored so that

$$
g(\theta)=(1-\cos \theta)^{n-m} A(\cos \theta),
$$

where $A(x)$ is a polynomial in $x$ of degree $b_{n}-n+m$.

Lemma 2. If $b_{1}, b_{2}, \ldots, b_{n}$ are odd integers, and if $n>m$, we have

$$
g^{\prime}(\theta)=\sin \theta\left(1-\cos ^{2} \theta\right)^{n-m-1} B(\cos \theta),
$$

where $B(x)$ is a polynomial of degree $b_{n}-2 n+2 m+1$.

Lemma 3 . Let

$$
P_{s}(x)=\int_{x}^{1}\left(1-t^{2}\right)^{n-1} t^{2 s} d t \quad \text { for } s=0,1,
$$

and

$$
Q(x, \zeta)=P_{1}(x)-\zeta^{2} P_{0}(x)
$$

The polynomial $Q(x, \zeta)$ is nonnegative in the interval $-1 \leq x \leq 1$ if, and only if,

$$
\zeta \leq z_{n} .
$$

Here $z_{n}$ is the root of the equation

$$
R(z) \equiv Q(-z, z) \equiv \int_{-z}^{1}\left(1-\mathrm{t}^{2}\right)^{n-1}\left(t^{2}-z^{2}\right) d t=0,
$$

which falls into the interval $(0,1)$.

$$
\text { Moreover, let } w_{n}=\sqrt{n+\frac{1}{2}} z_{n} .
$$

Then $\lim _{n \rightarrow \infty} w_{n}=\rho$ exists and is the root of the equation

$$
F(\rho)=\sqrt{ } \pi\left(\frac{1}{2}-\rho^{2}\right)-2 \rho^{3} \int_{0}^{1}\left(1-t^{2}\right) e^{-\rho^{2} t^{2}} d t=0
$$

which is located in the interval $\left(0, \sqrt{\frac{1}{2}}\right)$.

The proofs of the lemmas 1 and 2 are completely analogous to the proofs given for the corresponding lemmas in an earlier paper [3], in which nonnegative trigonometric polynomials of the form (6) were studied. Lemma 3 is identical with lemma 3 of [3]; we omit therefore the proofs of the three lemmas and refer instead to [3].

The function $g(\theta)$ as defined by (5) and (2a) depends on the parameters $b_{1}, \ldots, b_{n}, d_{1}, \ldots, d_{m}$. It is sometimes convenient to indicate this fact by writing $g(\theta)=g\left(\theta \mid b_{1}, \ldots, b_{n} ; d_{1}, \ldots, d_{m}\right)$. Similarly, we shall write occasionally $V_{0}=V_{0}\left(b_{1}, \ldots ., b_{n}\right)$ for the Vandermonde determinant formed from the numbers $b_{1}^{2}, \ldots, b_{n}^{2}$.

It is possible to obtain some of the results of our previous paper as particular cases of results obtained in the present paper. For this purpose we need the following lemma.

Lemma 4. Let $g\left(\theta \mid b_{1}, \ldots, b_{n} ; d_{1}, \ldots, d_{m}\right)$ be the function defined by $(5)$ and $(2 a)$. If we put $d_{v}=b_{\rho}$, we obtain

$$
\begin{aligned}
& \frac{g\left(\theta \mid b_{1}, \ldots, b_{n} ; d_{1}, \ldots, d_{v-1}, b_{\rho}, d_{v+1}, \ldots, d_{m}\right)}{V_{0}\left(b_{1}, \ldots, b_{n}\right)} \\
& \quad=\frac{g\left(\theta \mid b_{1}, \ldots, b_{\rho-1}, b_{\rho+1}, \ldots, b_{n} ; d_{1}, \ldots, d_{v-1}, d_{v+1}, \ldots, d_{m}\right)}{V_{0}\left(b_{1}, \ldots, b_{\rho-1}, b_{\rho+1}, \ldots, b_{n}\right)},
\end{aligned}
$$


that is, the substitution $d_{v}=b_{\rho}$ transforms the function

$$
\frac{g\left(\theta \mid b_{1}, \ldots, b_{n} ; d_{1}, \ldots, d_{m}\right)}{V_{0}\left(b_{1}, \ldots, b_{n}\right)}
$$

into a similar function that does not contain any more the parameters $d_{v}$ and $b_{\rho}$.

It is possible to give an elementary proof of (14) by a straightforward computation. The validity of (14) becomes apparent, however, if we remember that the rational function $\phi(t)$ is the Fourier transform of $C e^{-a x} g\left(x \mid b_{1}, \ldots, b_{n} ; d_{1}, \ldots, d_{m}\right)$.

\section{Nonnegative Trigonometric Polynomials}

In the following we assume that the $b_{1}, b_{2}, \ldots, b_{n}$ are positive integers, while the $d_{1}, \ldots, d_{m}$ may be any positive real numbers. The function $g(\theta)$, defined by (5) is then a trigonometric polynomial. According to condition (I) the function $\phi(t)$ is a characteristic function if, and only if, $g(\theta)$ is nonnegative. The purpose of this paper is to consider configurations that lead to nonnegative trigonometric polynomials. For this discussion it is convenient to introduce occasionally a new variable:

$$
x=\cos \theta,
$$

and to use the notation

$$
P(x)=g(\arccos x) .
$$

Since we assume from now on that the $b_{j}$ 's are integers, the substitution (15) transforms $\cos b_{j} \theta$ into $T_{b_{j}}(x)$, where $T_{b_{j}}(x)$ is the $b_{j}$ th Tchebycheff polynomial of the first kind. Therefore, we see from (5) that under the conditions of lemma 1 ,

$$
P(x)=\sum_{j=1}^{n}(-1)^{j-1} \Delta_{j}\left[1-\lambda_{j} T_{b_{j}}(x)\right]=(1-x)^{n-m} A(x) .
$$

In a similar manner, we have under the conditions of lemma 2,

$$
P^{\prime}(x)=\sum_{j=1}^{n}(-1)^{j} \Delta_{j} \lambda_{j} T_{b_{j}}^{\prime}(x)=-\left(1-x^{2}\right)^{n-m-1} B(x) .
$$

When we make this substitution we shall use the fact that the trigonometric polynomial $g(\theta)$ is nonnegative for all $\theta$ if, and only if, the polynomial $P(x) \geq 0$ for $|x| \leq 1$. From (17) we obtain immediately

Condition (III). The trigonometric polynomial $g(\theta)$ is nonnegative if, and only if, the polynomial $A(x)$, introduced by lemma 1 , is nonnegative for $|x| \leq 1$.

We use condition (III) and the lemmas obtained in section 2 to derive conditions for certain trigonometric polynomials. The results are contained in the subsequent statements.

Statement 1. If the $b_{j}$ are the first $n$ consecutive integers, $i . e ., b_{j}=j$ for $j=1,2, . ., n$, and if $m=1$, the trigonometric polynomial $g(\theta)$ is nonnegative if, and only if, $2 d_{1}^{2} \geq n$.

In this case we have $\lambda_{j} \equiv 1-j^{2} / d_{1}^{2}, j=1,2, \ldots, n$ and obtain from (17)

$$
P(x)=\sum_{j=1}^{n}(-1)^{j-1} \Delta_{j}\left[1-\left(1-\frac{j^{2}}{d_{1}^{2}}\right) T_{j}(x)\right]=(1-x)^{n-1}(a+b x) .
$$

From this equation it is possible to determine the coefficients $a$ and $b$. After some elementary computations we obtain

$$
P(x)=2^{n-1} \Delta_{n} \frac{\left[n(n-1)+d_{1}^{2}\right]}{d_{1}^{2}}(1-x)^{n-1}\left\{1+\frac{n^{2}-d_{1}^{2}}{n(n-1)+d_{1}^{2}} x\right\} .
$$


Clearly, $P(x) \geq 0$ for $|x| \leq 1$ if, and only if, $\left|n^{2}-d_{1}^{2}\right| \leq n(n-1)+d_{1}^{2}$. This inequality can be written as $-n^{2}+n-d_{1}^{2} \leq n^{2}-d_{1}^{2} \leq n^{2}-n+d_{1}^{2}$. Since $n$ is a positive integer, this condition reduces to $2 d_{1}^{2} \geq n$.

Corollary to statement 1 . If the $b$, are the first $n$ consecutive even integers, i. e., if $b_{j}=2 j$ for $j=1,2, \ldots, n$, and if $m=1$, then the trigonometric polynomials $g(\theta)$ is nonnegative if, and only if, $d_{1}^{2} \geq 2 n$. The corollary follows almost immediately from statement 1 .

Statement 2. If the $b_{j}$ are the first $n$ consecutive integers, $i$. e., if $b_{j}=j$ for $j=1,2, \ldots, n$, and if $m=2$, the trigonometric polynomial $g(\theta)$ is nonnegative, if, and only if, a certain polynomial $Q(x)$ is nonnegative for $|x| \leq 1$. Here

$$
Q(x)=L\left(n, d_{1}, d_{2}\right) x^{2}+M\left(n, d_{1}, d_{2}\right) x+N\left(n, d_{1}, d_{2}\right),
$$

where

$$
\left.\begin{array}{l}
L\left(n, d_{1}, d_{2}\right)=n^{4}-n^{2}\left(d_{1}^{2}+d_{2}^{2}\right)+d_{1}^{2} d_{2}^{2}, \\
M\left(n, d_{1}, d_{2}\right)=2 n^{4}-6 n^{3}+4 n^{2}-n+n\left(d_{1}^{2}+d_{2}^{2}\right)-2 d_{1}^{2} d_{2}^{2}, \\
N\left(n, d_{1}, d_{2}\right)=n^{4}-6 n^{3}+7 n^{2}-2 n+\left(n^{2}-n\right)\left(d_{1}^{2}+d_{2}^{2}\right)+d_{1}^{2} d_{2}^{2} .
\end{array}\right\}
$$

Proof. From (2a) we see that

$$
\lambda_{j}=\left(1-\frac{j^{2}}{d_{1}^{2}}\right)\left(1-\frac{j^{2}}{d_{2}^{2}}\right)
$$

for $j=1,2, \ldots$, and obtain from (17)

$$
P(x)=\sum_{j=1}^{n}(-1)^{j-1} \Delta_{j}\left\{1-\left(1-\frac{j^{2}}{d_{1}^{2}}\right)\left(1-\frac{j^{2}}{d_{2}^{2}}\right) T_{j}(x)\right\}=(1-x)^{n-2}\left(a x^{2}+b x+c\right) .
$$

From this equation it is possible to determine the coefficients $a, b$, and $c$. After some elementary computation, one obtains

$$
P(x)=\frac{2^{n-1} \Delta_{n}}{d_{1}^{2} d_{2}^{2}}(1-x)^{n-2} Q(x) .
$$

A particular case can be discussed easily. If $d_{1}=(n+1)$ and $d_{2}=(n+2)$, we obtain from (20) $L=4(n+1)(2 n+1), M=-4(2 n+1)\left(2 n^{2}+n+2\right), N=4 n^{4}+4 n^{3}+19 n^{2}+5 n+4 . \quad$ It is then easy to show that $Q(x)$ is positive in $-1 \leq x \leq+1$ so that $P(x)$ is nonnegative for the values $k_{j}=j(j=1,2, \ldots, n)$ and $m=2, d_{1}=(n+1), d_{2}=(n+2)$. This means in probabilistic language that the rational function with $2 n+1$ poles $-i(a \pm i j)(j=1, \ldots, n)$ and $-i a$ and the four zeros $-i\left(a \pm i d_{1}\right),-i\left(a \pm i d_{2}\right)$ is a characteristic function.

Statement 3. If the $b_{j}$ are the first $n$ consecutive odd integers, $i$. e., if $b_{j}=2 j-1$ for $j=1,2, \ldots, n$, and if $m=1$ while $n>1^{4}$, then the trigonometric polynomial $g(\theta)$ is nonnegative if either one of the following two conditions is satisfied

$$
\begin{aligned}
& d \geq \sqrt{2 n-1}, \\
& d<\sqrt{2 n-1},
\end{aligned}
$$

but

$$
\sqrt{\frac{(2 n-1)-d^{2}}{(2 n-1)^{2}-d^{2}}} \leq z_{n-1}
$$

${ }^{4}$ The case $n=m=1$ is covered by statement 1 , and can therefore be excluded here. 
where $z_{n-1}$ is the root of the equation

$$
\int_{-z}^{1}\left(1-t^{2}\right)^{n-2}\left(t^{2}-z^{2}\right) d t=0
$$

which falls into the interval $(0,1)$.

If neither (i) nor (ii) is satisfied, then the function $g(\theta)$ assumes also negative values.

Corollary to statement 3 . If for large $n$ and $d<2 n-1$

$$
\sqrt{\frac{\left(n+\frac{1}{2}\right)\left[(2 n-1)-d^{2}\right]}{(2 n-1)^{2}-d^{2}}} \leq \rho=0.5939157 \ldots
$$

then $g(\theta)$ is nonnegative.

In formulating this statement, we wrote for $d_{1}$ simply $d$. From $(2 a)$ we see that

$$
\lambda_{j}=1-\frac{(2 j-1)^{2}}{d^{2}} \text { for } j=1,2, \ldots, n .
$$

According to lemma 2, as expressed by (18), we have

$$
P^{\prime}(x)=\frac{1}{d^{2}} \sum_{j=1}^{n}(-1)^{j}\left[d^{2}-(2 j-1)^{2}\right] \Delta_{j} T_{2 j-1}^{\prime}(x)=-\left(1-x^{2}\right)^{n-2} B(x),
$$

where $B(x)$ is a polynomial of the second degree. From this equation it is possible to determine $B(x)$, we obtain

$$
B(x)=\gamma\left\{\left[1-\frac{2 n-1}{d^{2}}\right]-\left[1-\frac{(2 n-1)^{2}}{d^{2}}\right] x^{2}\right\},
$$

where

$$
\gamma=2^{2 n-2}(2 n-1) \Delta_{n}>0 .
$$

From (21) we see that

$$
\left.\begin{array}{rl}
B(-1) & =B(1)=\frac{2(2 n-1)(n-1)}{d^{2}} \gamma>0 \\
B(0) & =\left[1-\frac{2 n-1}{d^{2}}\right] \gamma>0 \\
B^{\prime}(x) & =-2 \gamma\left[1-\frac{(2 n-1)^{2}}{d^{2}}\right] x ; \quad B^{\prime \prime}(x)=-2 \gamma\left[1-\frac{(2 n-1)^{2}}{d^{2}}\right]
\end{array}\right\} .
$$

We first assume $d \geq 2 n-1$, then $B^{\prime}(x) \leq 0$ for $0 \leq x \leq 1$. Since $B(1)>0$, we have $B(x)>0$ in the interval $(0,1)$, from $B(-x)=B(x)$ it is seen that $B(x)>0$ for $|x| \leq 1$. Next we suppose $(2 n-1)>d \geq \sqrt{2 n-1}$, then $B(x)$ has a minimum at the point $x=0$, so that $B(x) \geq B(0)>0$. Summarizing, we see that $B(x) \geq 0$ for $|x| \leq 1$, provided $d \geq \sqrt{2 n-1}$. But this means that $P^{\prime}(x) \leq 0$ in $|x| \leq 1$. From (17) it is seen that $P(1)=0$ if $n>m$, so that $P(x)>0$ in $|x| \leq 1$, and therefore $g(\theta) \geq 0$ for all $\theta$, so that condition (i) is proved.

We next consider the case $d<\sqrt{2 n-1}$. We write

$$
B(x)=\left[\frac{(2 n-1)^{2}}{d^{2}}-1\right] \gamma\left\{x^{2}-\frac{(2 n-1)-d^{2}}{(2 n-1)^{2}-d^{2}}\right\}
$$


If we denote by

and by

$$
\gamma^{\prime}=\left[\frac{(2 n-1)^{2}}{d^{2}}-1\right] \gamma>0
$$

then we have

$$
\zeta^{2}=\frac{(2 n-1)-d^{2}}{(2 n-1)^{2}-d^{2}}>0
$$

Therefore,

$$
B(x)=\gamma^{\prime}\left(x^{2}-\xi^{2}\right) \text { and } \quad P^{\prime}(x)=-\gamma^{\prime}\left(1-x^{2}\right)^{n-2}\left(x^{2}-\xi^{2}\right) \text {. }
$$

$$
P(x)=\gamma^{\prime} \int_{x}^{1}\left(1-t^{2}\right)^{n-2}\left(t^{2}-\gamma^{2}\right) d t .
$$

Condition (ii) and the corollary to statement 3 follow then immediately from lemma 3 ; a brief table of the $z_{n}$ is contained [3].

We proceed to discuss a more complicated case by assuming that the rational function $\phi(t)$ has two zeros (i. e., $m=2$ ) and that it has the first $n$ consecutive odd integers as poles, so that $b_{j}=2 j-1$ for $j=1,2, \ldots, n$.

We obtain then from (18)

where

$$
P^{\prime}(x)=-\gamma\left(1-x^{2}\right)^{n-3}\left[\mathrm{~A}+C x^{2}+D x^{4}\right],
$$

We write ${ }^{5}$

$$
\left.\begin{array}{l}
\gamma=2^{2 n-6}(2 n-1) \Delta_{n} \\
D=16 \lambda_{n} \\
C=4\left[(2 n-9) \lambda_{n}-(2 n-1) \lambda_{n-1}\right] \\
A=\left(2 n^{2}-13 n+22\right) \lambda_{n}-(2 n-1)(2 n-7) \lambda_{n-1}+(n-1)(2 n-1) \lambda_{n-2}
\end{array}\right\}
$$

so that

$$
a=\frac{C}{4 \lambda_{n}}, \quad b=-\frac{A}{4 \lambda_{n}}
$$

$$
P^{\prime}(x)=-4 \gamma \lambda_{n}\left(1-x^{2}\right)^{n-3}\left(4 x^{4}+a x^{2}-b\right) .
$$

For $n>m=2$ we have $P(1)=0$, and therefore ${ }^{6}$

$$
P(x)=4 \gamma \lambda_{n} \int_{x}^{1}\left(1-t^{2}\right)^{n-3}\left(4 t^{4}+a t^{2}-b\right) d t \quad \text { for } n \geq 3 .
$$

The nonnegativity of the polynomial $P(x)$ depends on the nature of the roots of the polynomial $B(x)=4 x^{4}+a x^{2}-b$. This introduces the need to distinguish a number of cases, for this purpose we write $h(\tau)=4 \tau^{2}+a \tau-b=4\left(\tau-\tau_{1}\right)\left(\tau-\tau_{2}\right)$, where

$$
\left.\begin{array}{rl}
\tau_{1} & =\left(-a-\sqrt{a^{2}+16 b}\right) / 8 \\
\tau_{2} & =\left(-a+\sqrt{a^{2}+16 b}\right) / 8 \\
B(x) & =h\left(x^{2}\right)
\end{array}\right\} .
$$

In the following we denote by

$$
R_{i}(z)=\int_{-}^{1}\left(1-t^{2}\right)^{n-3} t^{2(i-1)}\left(t^{2}-z^{2}\right) d t \text { for } i=1,2 .
$$

We are now in a position to formulate statement 4.

\footnotetext{
${ }^{5}$ We assume here $\lambda_{n} \neq 0$. We need not consider the case $\lambda_{n}=0$, since this reduces by lemma 4 to the case covered by statement 3 .

${ }^{6}$ If $n=m$, lemma 2 is not valid, and moreover, $P(1)>0$. The case $n=m=2$ would therefore require a separate discussion.
} 
Statement 4. If the $b_{j}$ are the first $n$ consecutive odd integers, that is, if $b_{j}=2 j-1$ for $j=1,2, \ldots, n$, and if $n>m=2$, then the trigonometric polynomial $g(\theta)$ is nonnegative if, and only if, one of the following nine, mutually exclusive, conditions is satisfied

(iv) $\quad \lambda_{n}>0,0>a>-4,4+a>b>-a^{2} / 16$, and $R_{2}\left(\sqrt{ } \tau_{2}\right)-\tau_{1} R_{1}\left(\sqrt{ } \tau_{2}\right) \geq 0$; if $b<0$, we have the additional condition $R_{2}\left(-\sqrt{ } \tau_{1}\right)-\tau_{2} R_{1}\left(-\sqrt{ } \tau_{1}\right) \geq 0$

$$
\lambda_{n}>0 \geq a+8>a+4 \geq b>-a^{2} / 16
$$

$\lambda_{n}>0,-4 \geq a>-8,0 \geq a+4 \geq b>-\mathrm{a}^{2} / 16$, and also $R_{2}\left(\sqrt{ } \tau_{2}\right)-\tau_{1} R_{1}\left(\sqrt{ } \tau_{2}\right) \geq 0$; if $b \neq 0$, we have the additional condition $R_{2}\left(-\sqrt{ } \tau_{1}\right)-\tau_{2} R_{1}\left(-\sqrt{ } \tau_{1}\right) \geq 0$

$$
\lambda_{n}<0, a \geq 0 \text {, and } b \geq a+4
$$

$$
\lambda_{n}<0, a<0 \text {, and } b \geq \max [0, a+4]
$$

$$
\lambda_{n}<0, a+4 \leq b<0 \text {, and } R_{2}\left(\sqrt{ } \tau_{1}\right)-\tau_{2} R_{1}\left(\sqrt{ } \tau_{1}\right) \leq 0 \text {. }
$$

The proof of this statement is rather tedious. It is, however, similar to the proof of statement 3 of the present paper and of statement $(\mathrm{F})$ of [3] and is therefore omitted.

We conclude this section by using lemma 4 to show that the earlier results may be obtained as particular cases of the statements of the present paper. We consider as an example statement 1 and write $n=s+1$ and put $d_{1}=k$, where $k$ is an integer. From lemma 4 we see that $g(\theta \mid 1, \ldots, s+1 ; k)$ and $g_{0}(\theta \mid 1, \ldots, k-1, k+1, \ldots, s+1)$ are simultaneously nonnegative so that statement 1 reduces to statement $\mathrm{C}$ of [3].

\section{References}

[1] E. Lukacs and O. Szász, Certain Fourier transforms of distributions, Can. J. Math. 3, 140-144 (1951).

[2] E. Lukacs and O. Szász, Analytic characteristic functions, Pac. J. Math. II, 615-625 (December 1952).

[3] E. Lukacs and O. Szász, Some nonnegative trigonometric polynomials connected with a problem in probability, J. Research NBS 48, 139-146 (1952) RP2297.

Washington, December 19, 1952. 